GUIDELINE UPDATE

\title{
Managing passengers with stable respiratory disease planning air travel: British Thoracic Society recommendations
}

\author{
Dinesh Shrikrishna, ${ }^{1}$ Robina K Coker, ${ }^{2}$ on behalf of the Air Travel Working Party of the \\ British Thoracic Society Standards of Care Committee
}

${ }^{1}$ National Heart \& Lung Institute, Royal Brompton Hospital and Imperial College London, UK ${ }^{2}$ National Heart \& Lung Institute, Hammersmith Hospital and Imperial College London, UK

Correspondence to

Dr Dinesh Shrikrishna, Royal Brompton Hospital, Department of Respiratory Medicine, London SW3 6NP, UK;

dinesh.shrikrishna@nhs.net

Accepted 27 June 2011

Published Online First

31 July 2011

\section{ABSTRACT}

This article summarises the key points from the 2011 British Thoracic Society (BTS) recommendations on managing passengers with respiratory disease planning air travel. The guidance aims to provide practical advice for respiratory specialists in secondary care and serves as a valuable reference for other healthcare professionals managing these patients. A greater awareness of the challenges posed by air travel will allow improved clinical assessment and practical advice to encourage patients to fly safely wherever possible.

\section{NEED FOR NEW RECOMMENDATIONS FOR PASSENGERS WITH RESPIRATORY DISEASE PLANNING AIR TRAVEL}

The question of how best to assess and advise on the safety of passengers with lung disease planning air travel is an increasingly common one faced by respiratory physicians and other healthcare professionals. Since the first BTS recommendations published in $2002^{1}$ and web updated in $2004,{ }^{2}$ a number of studies have confirmed that neither resting sea level oxygen saturations nor forced expiratory volume in $1 \mathrm{~s}\left(\mathrm{FEV}_{1}\right)$ reliably predict hypoxaemia, or complications of air travel, in passengers with respiratory disease. ${ }^{3-5}$ It has become clear that no reliable threshold in these variables can accurately determine the safety of air travel or the need for in-flight oxygen. Despite this, a North American service offering radio link assistance for in-flight medical emergencies logs $>17000$ calls a year, with respiratory events accounting for $10-12 \%$ of such calls from 2004 to 2008 (personal communication Dr Paulo Alves, MedAire, 2009). Therefore, physicians must be aware of the potential effects of the flight environment in those with lung disease, and the need for practical recommendations remains.

The 2011 guidance document now covers bronchiectasis, cancer, obesity, hyperventilation and dysfunctional breathing, pulmonary arteriovenous malformations and sinus and middle ear disease; and has expanded sections on infection and co-morbidity with cardiac disease. The recommendations are an expert consensus view based on literature reviews and aim to provide practical advice for respiratory specialists in secondary care, as well as providing a valuable reference for other healthcare professionals. The advice applies to commercial flights only (including scheduled repatriation with a medical or nurse escort), and excludes emergency aeromedical evacuations. However, if medical practitioners do assist at an in-flight medical emergency, most airlines will indemnify them, the aircraft will have medical equipment and they can often access specialist advice from ground-based support.

\section{PURPOSE OF RECOMMENDATIONS}

- To enhance safety for passengers with lung disease travelling on commercial flights and reduce respiratory complications.

- To promote further understanding among healthcare professionals that patients with respiratory disease may require clinical assessment and advice before air travel.

- To provide an authoritative up-to-date literature review of the latest available evidence.

- To provide consistent, practical and comprehensive advice for healthcare professionals managing these patients.

- To formulate key research questions to provoke further investigation, thereby providing a strengthened high quality evidence base for future guidelines.

- To promote the development of methods for monitoring the size of the problem.

A summary of key points from the new recommendations (table 1) and an algorithm for managing adult passengers with respiratory disease planning air travel (figure 1) are included here for practical reference in the clinic setting.

\section{Competing interests None.}

Provenance and peer review Not commissioned; internally peer reviewed.

\section{REFERENCES}

1. Coker RK, Boldy DA, Buchdahl R, et al. Managing passengers with respiratory disease planning air travel: British Thoracic Society recommendations. Thorax 2002;57:289-304.

2. Coker RK, Boldy DA, Buchdahl R, et al. Managing passengers with respiratory disease planning air travel: British Thoracic Society recommendations. 2004. http://www.brit-thoracic.org.uk.

3. Christensen CC, Ryg M, Refvem OK, et al. Development of severe hypoxaemia in chronic obstructive pulmonary disease patients at 2,438m (8,000ft) altitude. Eur Respir J 2000;15:635-9.

4. Coker RK, Shiner RJ, Partridge MR. Is air travel safe for those with lung disease? Eur Respir J 2007;30:1057-63.

5. Akero A, Christensen CC, Edvardsen A, et al. Pulse oximetry in the preflight evaluation of patients with chronic obstructive pulmonary disease. Aviat Space Environ Med 2008;79:518-24. 
Table 1 Summary of key points and recommendations

The flight environment and effects of altitude

- At cabin altitudes of 8000 feet the partial pressure of oxygen falls to the equivalent of breathing $15.1 \% \mathrm{O}_{2}$ at sea level

- In healthy passengers arterial oxygen tension $\left(\mathrm{PaO}_{2}\right)$ falls to $8.0-10 \mathrm{kPa}\left(60-75 \mathrm{mmHg}, \mathrm{SpO}_{2} 89-94 \%\right)$ and altitude exposure may worsen pre-existing hypoxaemia in patients with respiratory disease

- Neither resting sea level $\mathrm{SpO}_{2}$ nor $\mathrm{FEV}_{1}$ appear to predict hypoxaemia or complications accurately during or after air travel in patients with respiratory disease

Pre-flight assessment for adults

- Physicians should consider the patient's previous flight experience, flight duration and time since last exacerbation

- Usual care should be optimised with further evaluation (see algorithm - fig 1) in patient groups at greatest risk including:

- patients with previous air travel intolerance with significant respiratory symptoms (dyspnoea, chest pain, confusion or syncope)

- $\quad$ severe COPD ( $\mathrm{FEV}_{1}<30 \%$ pred), bullous lung disease, asthma, cystic fibrosis or pulmonary tuberculosis

- $\quad$ severe restrictive disease $(\mathrm{VC}<1 \mathrm{~L})$, including chest wall and respiratory muscle disease, especially with hypoxaemia and/or hypercapnia

- co-morbidity with conditions worsened by hypoxaemia (cerebrovascular disease, cardiac disease or pulmonary hypertension)

- recent pneumothorax or within six weeks of hospital discharge for acute respiratory illness

- risk of or previous venous thromboembolism

- pre-existing requirement for oxygen, CPAP or ventilator support

Contraindications to commercial air travel are infectious tuberculosis, ongoing pneumothorax with persistent air leak, major haemoptysis and a usual oxygen requirement at sea level at a flow rate exceeding $4 \mathrm{~L} / \mathrm{min}$

\section{Hypoxic challenge testing (HCT)}

- The sole purpose of this test is to determine whether a patient requires in-flight oxygen, and the physician should emphasise that it is not a 'fitness to fly' test

- $\mathrm{HCT}$ should be considered in patients with a sea level $\mathrm{SpO}_{2}<95 \%$ who are in one of the risk groups listed above and who do not already receive LTOT at sea level

- Patients already receiving LTOT at sea level should be prescribed in-flight oxygen at double their usual flow rate while at cruising altitude and should not require HCT

- Results of hypoxic challenge test $\left(15 \% \mathrm{FiO}_{2}\right.$ for 20 minutes) with revised SIGN grading:

Hypoxic challenge test result

- $\mathrm{PaO}_{2} \geq 6.6 \mathrm{kPa}(>50 \mathrm{mmHg}$ ) or $\mathrm{SpO} 2 \geq 85 \%$

- $\mathrm{PaO}_{2}<6.6 \mathrm{kPa}(<50 \mathrm{mmHg})$ or $\mathrm{SpO} 2<85 \%$

\section{Recommendation}

In-flight oxygen not required (C)

In-flight oxygen required at $2 \mathrm{~L} / \mathrm{min}$ via nasal cannulae (C)

\section{Venous thromboembolic disease (VTE)}

- The risk of VTE is greatest on flights lasting over eight hours and is reduced if patients occupy an aisle seat

- Low risk for VTE - all patients not in categories below

- Patients should avoid excess alcohol and caffeine-containing drinks, and be advised to remain mobile and/or exercise their legs during the flight (D)

- Moderate risk for VTE - family history of VTE, past history of VTE with identifiable cause, thrombophilia, obesity $\left(\mathrm{BMI}>30 \mathrm{~kg} / \mathrm{m}^{2}\right)$, height $>1.90 \mathrm{~m}$ or $<1.60 \mathrm{~m}$, significant medical illness in previous 6 weeks, cardiac disease, immobility, pregnancy or oestrogen therapy (HRT \& some oral contraceptives) and post-natal within 2 weeks of delivery

- Advice as per above - patients should wear below knee elastic compression stockings and not use sedatives or sleep for prolonged periods in abnormal positions (D)

- High risk for VTE - past history of idiopathic VTE, within six weeks of major surgery or trauma, active malignancy

- Advice as per above - no evidence to support the use of low or high dose aspirin

- Consider pre-flight prophylactic dose of low molecular weight heparin or formal anticoagulation (D)

- Patients who have had a VTE should be advised not to travel for four weeks or until proximal deep vein thrombosis treated and symptoms resolved, with no evidence of pre- or post-exercise desaturation (D)

\section{Additional notes}

- Bronchodilator inhalers, with spacer, should be carried in hand luggage for acute exacerbations of asthma or COPD

- Advise on emergency supply of antibiotics and/or steroids for severe asthma, COPD and interstitial lung disease

- In-flight oxygen is prescribed at a rate of $2 \mathrm{~L}$ or $4 \mathrm{~L} / \mathrm{min}$ via nasal cannulae and is usually supplied by the airline with advance booking. Patients may use their own oxygen cylinders or portable concentrators at the airline's discretion.

- Dry cell battery-powered continuous positive airway pressure (CPAP) machines can be used (except during take-off/landing)

- Further disease specific recommendations and paediatric guidance are detailed in the full document. 
Figure 1 Algorithm for managing adult passengers with respiratory disease planning air travel. LTOT, longterm oxygen therapy; VTE, venous thromboembolism.

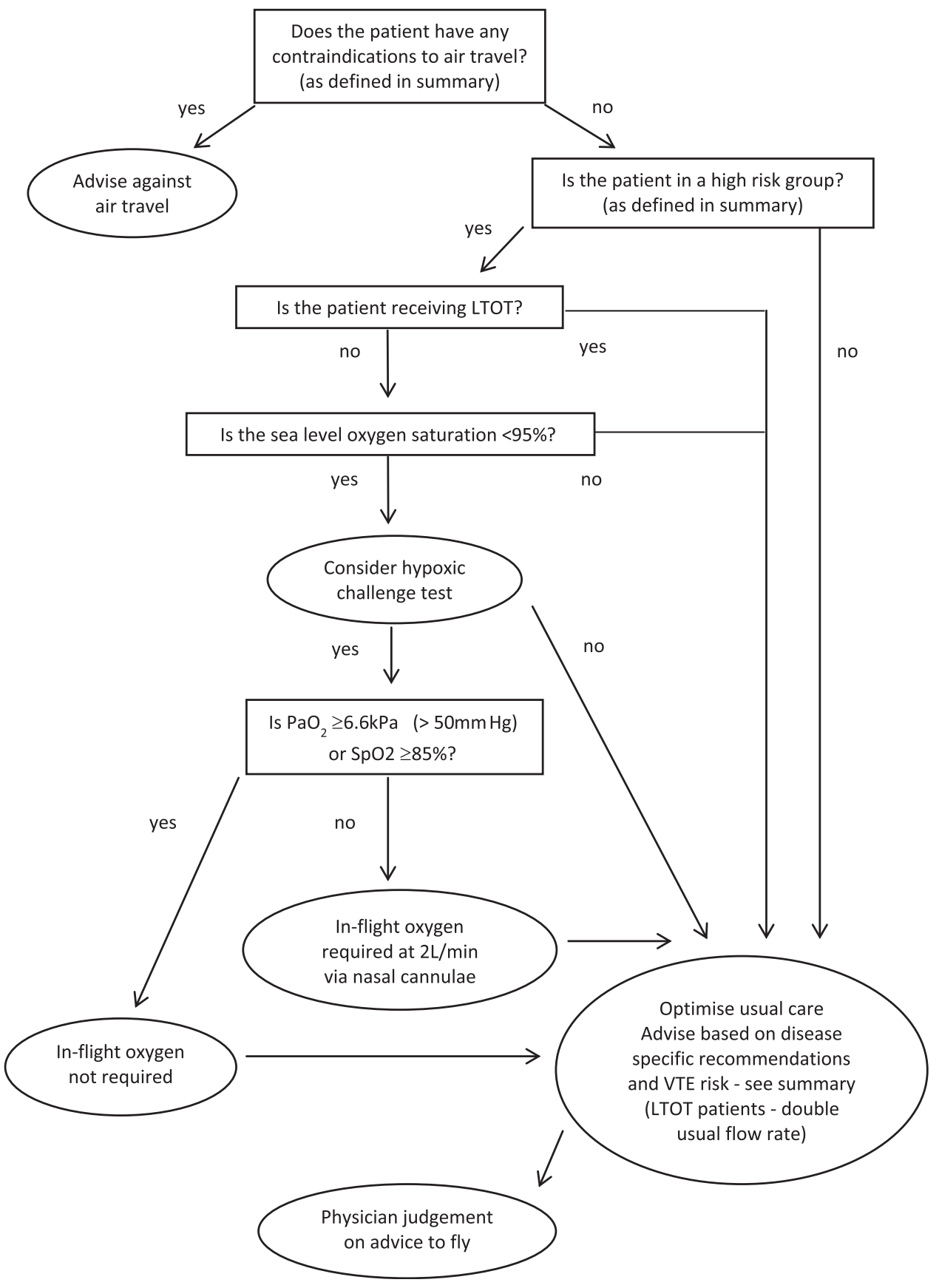

